\title{
Complex periodic potentials with real band spectra
}

\author{
Carl M. Bender ${ }^{1}$, Gerald V. Dunne ${ }^{2}$, and Peter N. Meisinger ${ }^{1}$ \\ ${ }^{1}$ Department of Physics, Washington University, St. Louis MO 63130, USA \\ ${ }^{2}$ Department of Physics, University of Connecticut, Storrs CT 06269, USA
}

(February 1, 2008)

\begin{abstract}
This paper demonstrates that complex $\mathcal{P} \mathcal{T}$-symmetric periodic potentials possess real band spectra. However, there are significant qualitative differences in the band structure for these potentials when compared with conventional real periodic potentials. For example, while the potentials $V(x)=i \sin ^{2 N+1}(x)(N=0,1,2, \ldots)$ have infinitely many gaps, at the band edges there are periodic wave functions but no antiperiodic wave functions. Numerical analysis and higher-order WKB techniques are used to establish these results.
\end{abstract}

For a quantum mechanical model having a periodic potential the Schrödinger equation is

$$
-\psi^{\prime \prime}(x)+V(x) \psi(x)=E \psi(x)
$$

where the potential $V(x)$ is periodic with period $P$ :

$$
V(x+P)=V(x) .
$$

In conventional treatments of Eq. (11) [1,2] the periodic potential $V(x)$ is assumed to be real. Imposing the condition that the wave function $\psi(x)$ be bounded leads to a real spectrum consisting of continuous bands separated by gaps. There is an infinite number of bands and gaps, except for the special family of so-called finite-gap potentials such as the Lamé potentials [3].

In this note we extend the conventional analysis to include the case of complex periodic potentials. We find that complex periodic potentials having $\mathcal{P} \mathcal{T}$ symmetry exhibit real band spectra, despite the non-Hermitian character of the Schrödinger equation (11). (Here, $\mathcal{P}$ represents parity reflection and $\mathcal{T}$ represents time reversal.) Potentials having this symmetry satisfy

$$
[V(-x)]^{*}=V(x) .
$$

Examples of such potentials are $i \sin x, i \sin ^{3}(x)$, and $e^{i x}$. In addition to the property that these potentials have real spectra, the band structure displays several novel features that are strikingly different from the case of real periodic potentials.

The work reported here was motivated by recent investigations of non-Hermitian $\mathcal{P} \mathcal{T}$-symmetric Hamiltonian models having real discrete spectra. One such class of models is defined by the Hamiltonian 㓞

$$
H=p^{2}+x^{2}(i x)^{\epsilon} \quad(\epsilon \geq 0) .
$$

Despite the lack of conventional Hermiticity, the spectrum of this Hamiltonian is real, positive, and discrete; each of the energy levels increases as a function of increasing $\epsilon$. It has been observed that the reality of the spectrum is a consequence of $\mathcal{P} \mathcal{T}$ symmetry, which is a weaker condition than Hermiticity. This observation has also been used to construct new classes of quasi-exactly solvable quantum theories [5] and to study new kinds of symmetry breaking in quantum field theory [6.7]. There have been many other instances of non-Hermitian $\mathcal{P} \mathcal{T}$-invariant Hamiltonians in physics. Energies of solitons on a complex Toda lattice have been found to be real [8]. Hamiltonians rendered nonHermitian by an imaginary external field have been used to study population biology 9 and to study delocalization transitions such as vortex flux-line depinning in type-II superconductors [10]. In these last two cases, initially real eigenvalues bifurcate into the complex plane due to the increasing external field, indicating the growth of populations or the unbinding of vortices.

We begin by summarizing the standard Floquet analysis of the Schrödinger equation (11) for the case where $V(x)$ is real and periodic [2]. We define a fundamental pair of linearly independent solutions $u_{1}(x)$ and $u_{2}(x)$ satisfying the initial conditions

$$
\begin{array}{ll}
u_{1}(0)=1, & u_{1}^{\prime}(0)=0 \\
u_{2}(0)=0, & u_{2}^{\prime}(0)=1 .
\end{array}
$$

Any solution $\psi(x)$ to Eq. (11) is a linear combination of $u_{1}(x)$ and $u_{2}(x)$. It is then a straightforward algebraic exercise to show that $\psi(x)$ is bounded provided that the discriminant $\Delta(E)$, which is defined by 


$$
\Delta(E) \equiv u_{1}(P)+u_{2}^{\prime}(P)
$$

satisfies the constraint

$$
-2 \leq \Delta(E) \leq 2
$$

To illustrate the features of the discriminant we consider a typical periodic potential, $V(x)=\sin (x)$, for which the period $P=2 \pi$. In Fig. 1 1 we plot $\Delta(E)$ as a function of $E$. Note that $\Delta(E)$ is oscillatory and is well approximated by the function $2 \cos (\pi \sqrt{E})$ for large $E$. The crucial feature of $\Delta(E)$, which cannot be seen from this plot, is that its graph crosses the lines \pm 2 an infinite number of times; each of the maxima of $\Delta(E)$ lies above 2 and each of the minima lies below -2 . The regions of energy for which $|\Delta(E)| \leq 2$ are called bands and the regions of energy for which $|\Delta(E)|>2$ are called gaps. The gap size decreases exponentially as a function of $E$. The band edges at which $\Delta(E)=2$ correspond to periodic solutions to Eq. (1),$\psi(x)=\psi(x+P)$, and the band edges at which $\Delta(E)=-2$ correspond to antiperiodic solutions $\psi(x)=-\psi(x+P)$.

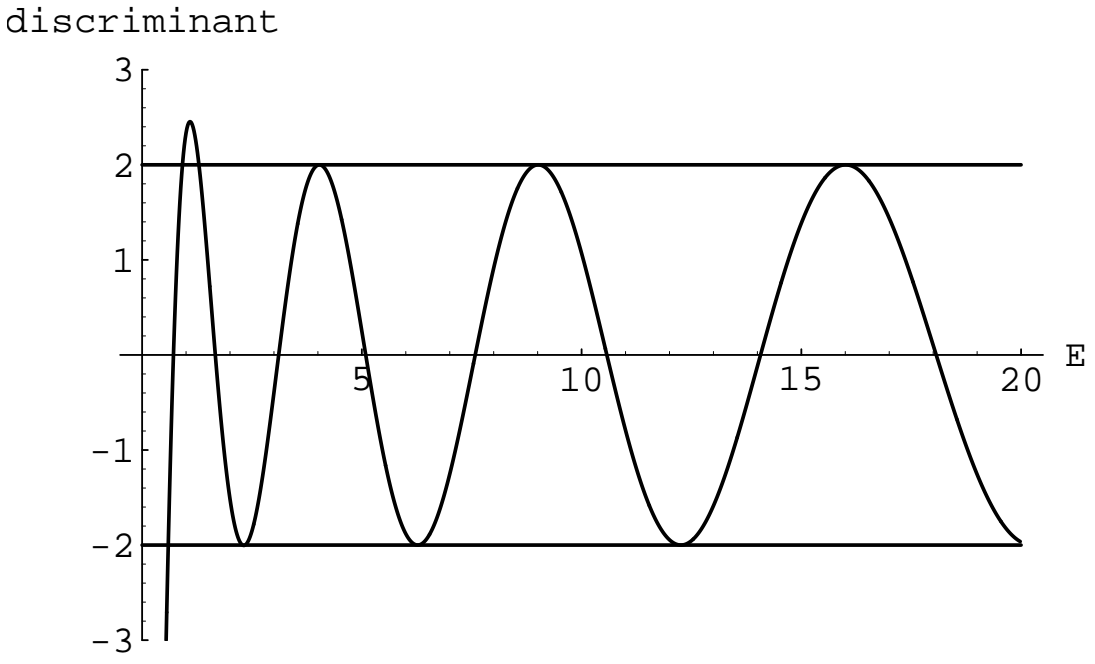

FIG. 1. The discriminant $\Delta(E)$ plotted as a function of $E$ for the real periodic potential $V(x)=\sin (x)$. Although it cannot be seen in the figure, all local maxima lie above the line $\Delta=2$ and all local minima lie below the line $\Delta=-2$. The regions of energy $E$ for which $|\Delta| \leq 2$ correspond to bands and the regions where $|\Delta|>2$ correspond to gaps. There are infinitely many gaps and these gaps become exponentially narrow as $E$ increases.

Now consider the calculation of the discriminant for the case of a complex periodic potential $V(x)$. In general, a complex periodic potential will have no bounded solutions because the discriminant is typically complex. However, for complex $\mathcal{P} \mathcal{T}$-symmetric periodic potentials, one can easily show that the discriminant $\Delta(E)$ is real when $E$ is real. The $\mathcal{P} \mathcal{T}$ symmetry is crucial here; for a potential that is not $\mathcal{P} \mathcal{T}$ symmetric [one that does not satisfy Eq. (3)], the discriminant is complex for all values of $E$. Having established that complex $\mathcal{P} \mathcal{T}$-symmetric periodic potentials have real discriminants, we can then apply the criterion in Eq. (7) to locate the real energy bands within which the corresponding wave function $\psi(x)$ is a bounded function.

We have computed the discriminants for the class of complex $\mathcal{P} \mathcal{T}$-symmetric periodic potentials

$$
V(x)=i \sin ^{2 N+1}(x) \quad(N=0,1,2, \ldots) .
$$

In Figs. 2 月 we plot the discriminants for the cases $N=0,1, \ldots, 5$. While these plots superficially resemble Fig. 1 for large $E$, they exhibit new and intriguing features that are significantly different from the case of a real periodic potential. The most obvious new feature is the appearance for $N \geq 1$ of a local minimum of $\Delta(E)$ between -2 and 2. Such a dip is rigorously forbidden in the case of real periodic potentials [2].

The most dramatic differences between the discriminants for the complex $\mathcal{P} \mathcal{T}$-symmetric periodic potentials in Eq. (8) and real periodic potentials cannot be easily seen in the figures. We have performed a careful numerical study of the local minima and maxima of $\Delta(E)$. Our study reveals that none of the local minima lies below -2 . This shows 
that there are no antiperiodic solutions $\psi(x)$ to the Schrödinger equation (1). Nevertheless, all of the local maxima of $\Delta(E)$ lie above 2 . Hence, there are an infinite number of band gaps in the spectrum and the band-edge wave functions are periodic.

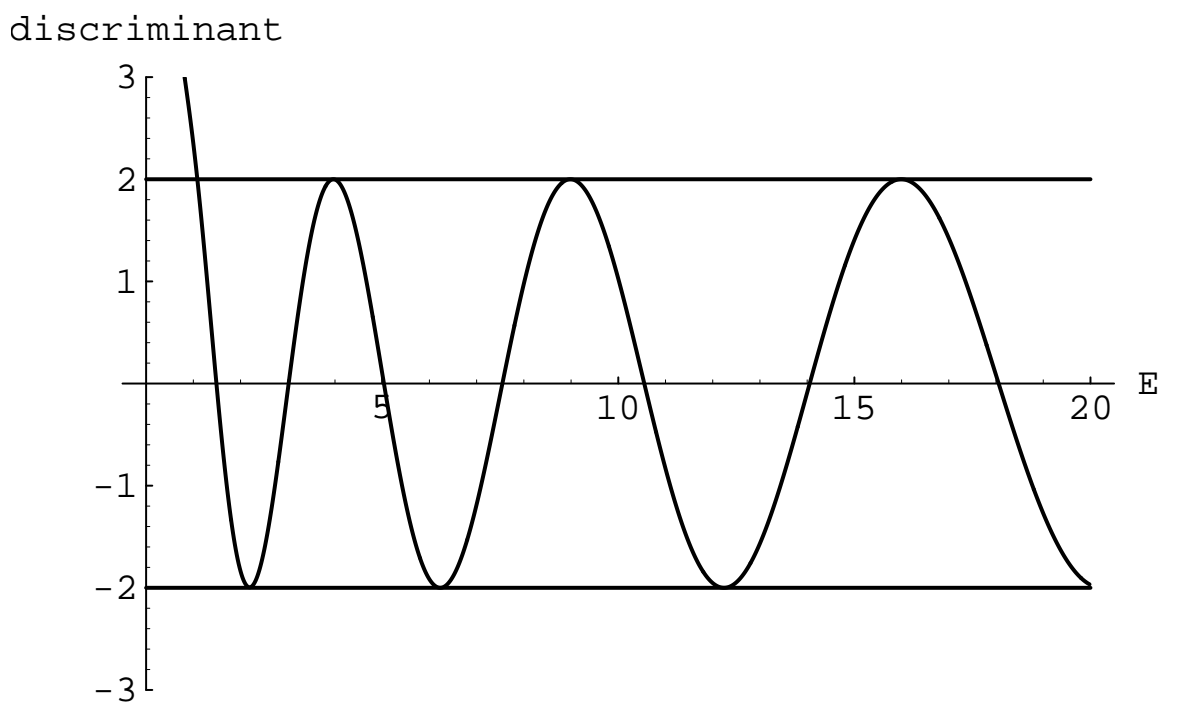

FIG. 2. The discriminant $\Delta(E)$ plotted as a function of $E$ for the complex periodic potential $V(x)=i \sin (x)$. On the scale of this figure for $E>2$ it is not possible to see any difference between this figure and Fig. 1. However, although it cannot be seen in this figure, all local maxima lie above the line $\Delta=2$ and all local minima lie above the line $\Delta=-2$. This behavior is distinctly different from the generic behavior in Fig. 1 exhibited by the real periodic potential $V(x)=\sin x$.

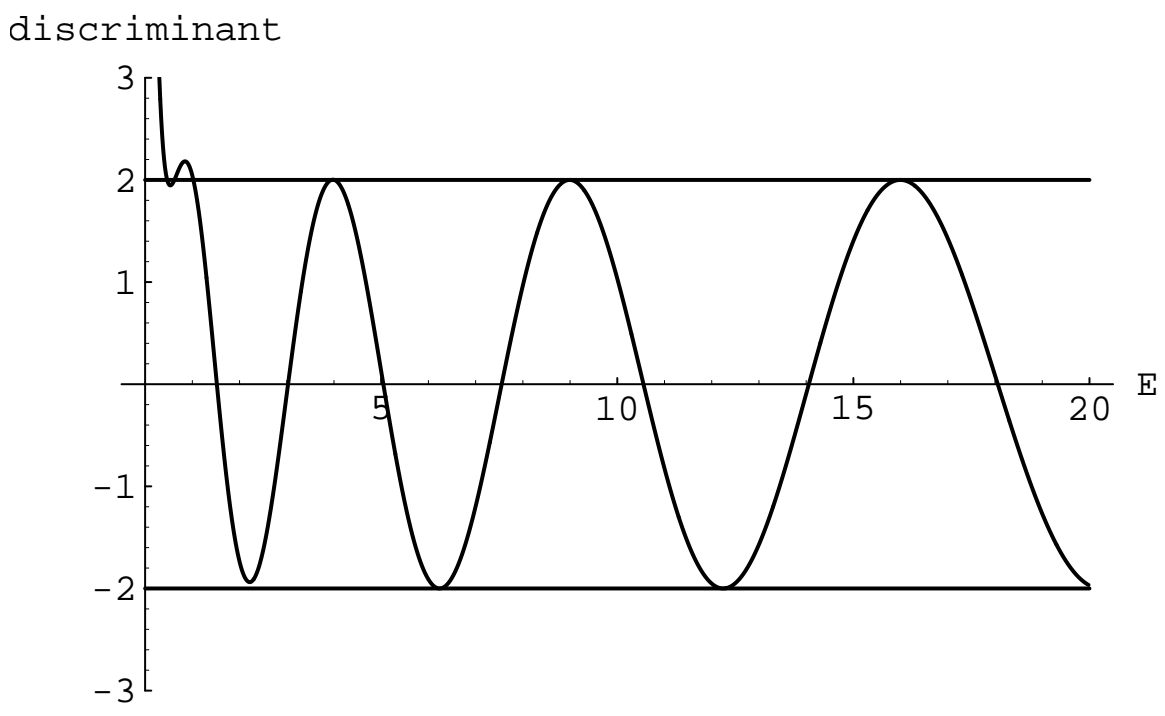

FIG. 3. The discriminant $\Delta(E)$ for the complex periodic potential $V(x)=i \sin ^{3}(x)$. All local maxima lie above the line $\Delta=2$ and all local minima lie above the line $\Delta=-2$. Note the appearance of a new local minimum near $E=0.5$. 


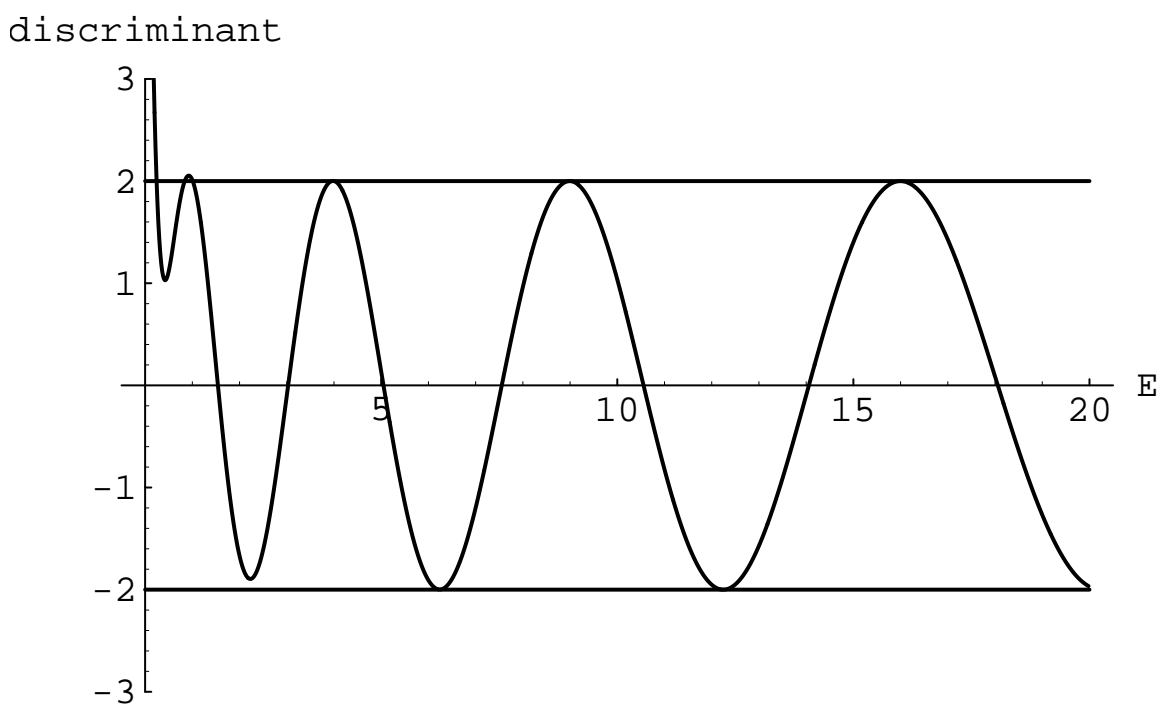

FIG. 4. The discriminant $\Delta(E)$ for the complex periodic potential $V(x)=i \sin ^{5}(x)$. All local maxima lie above the line $\Delta=2$ and all local minima lie above the line $\Delta=-2$.

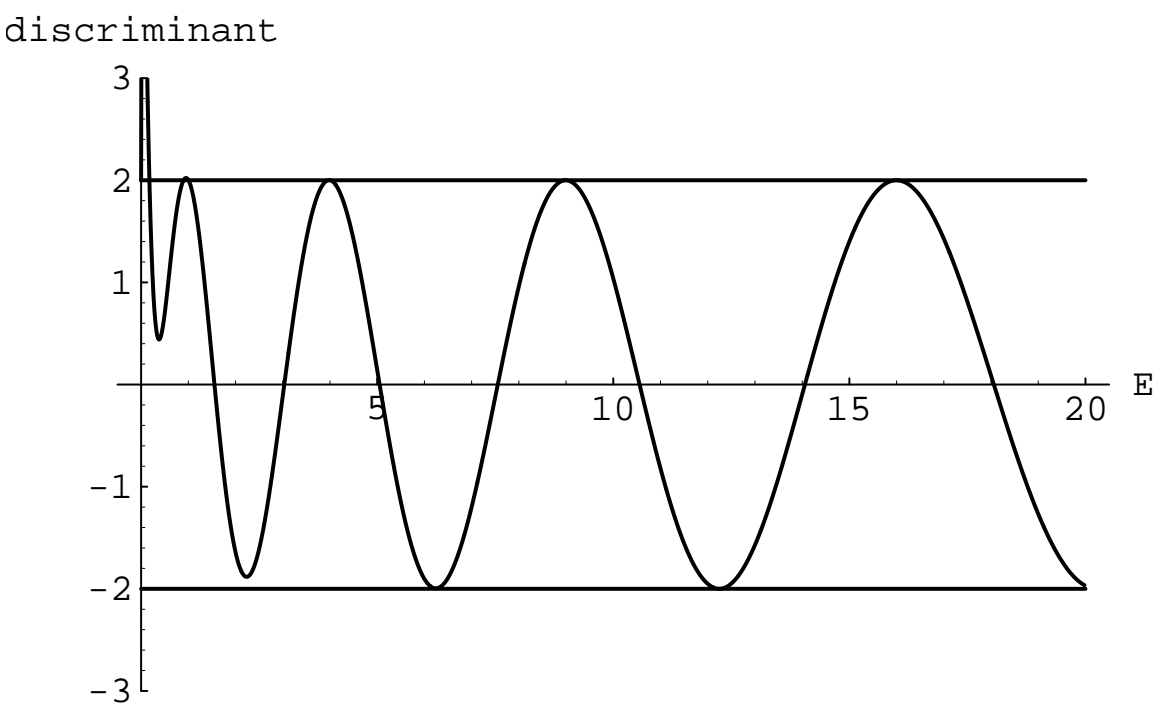

FIG. 5. The discriminant $\Delta(E)$ for the complex periodic potential $V(x)=i \sin ^{7}(x)$. All local maxima lie above the line $\Delta=2$ and all local minima lie above the line $\Delta=-2$. 


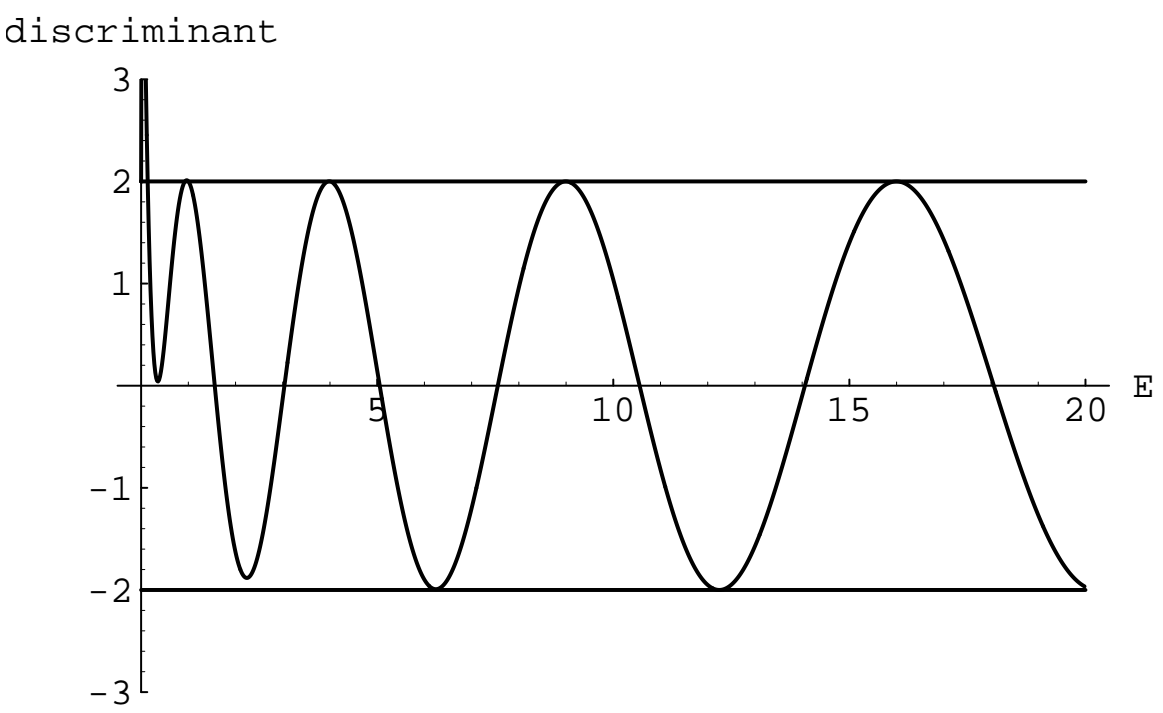

FIG. 6. The discriminant $\Delta(E)$ for the complex periodic potential $V(x)=i \sin ^{9}(x)$. All local maxima lie above the line $\Delta=2$ and all local minima lie above the line $\Delta=-2$.

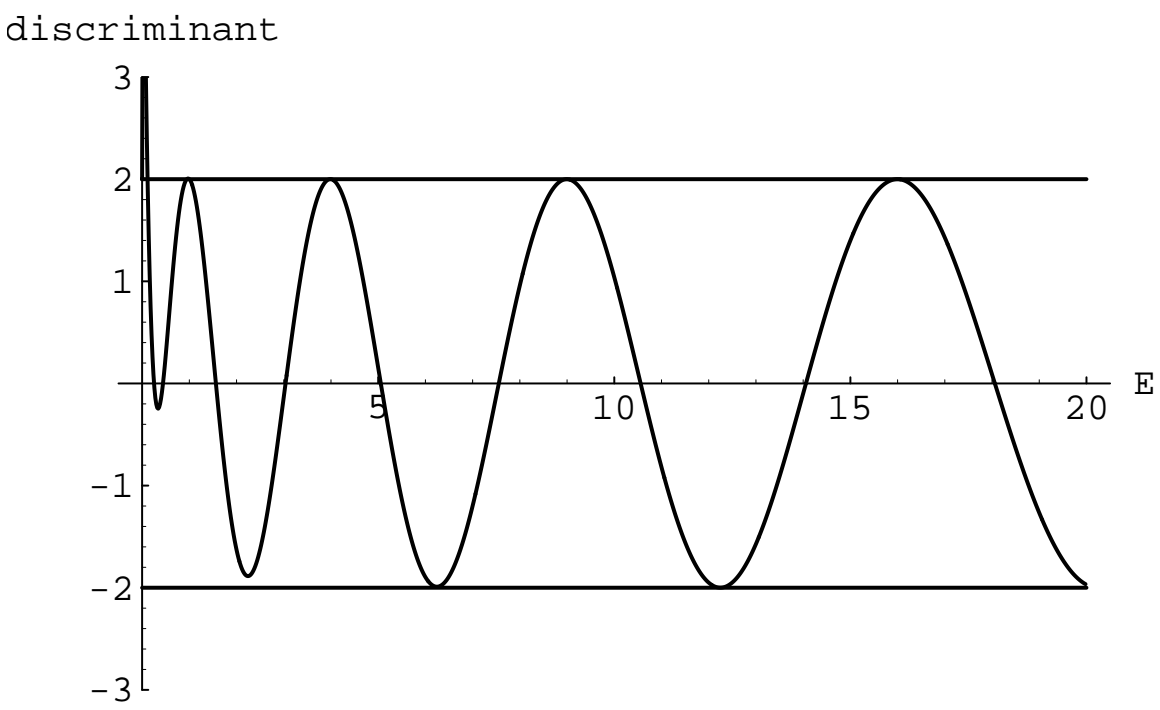

FIG. 7. The discriminant $\Delta(E)$ for the complex periodic potential $V(x)=i \sin ^{11}(x)$. All local maxima lie above the line $\Delta=2$ and all local minima lie above the line $\Delta=-2$.

To perform this numerical analysis it is necessary to locate the positions of the local minima and maxima of $\Delta(E)$ to extremely high accuracy. This can be done using WKB methods [11. We take the energy $E$ to be large $(E>>1)$ and define a small parameter $\epsilon$ by

$$
\epsilon=\frac{1}{\sqrt{E}}
$$

Then we make an exponential ansatz for the wave function $\psi(x)$ : 


$$
\psi(x)=\exp \left[\frac{i}{\epsilon} \sum_{n=0}^{\infty} \epsilon^{n} Q_{n}(x)\right] .
$$

Substituting $\psi(x)$ in Eq. (10) into the Schrödinger equation (11) gives a recursion relation for the functions $Q_{n}(x)$ :

$$
\begin{aligned}
1-\left[Q_{0}^{\prime}(x)\right]^{2} & =0, \\
i Q_{0}^{\prime \prime}(x)-2 Q_{0}^{\prime}(x) Q_{1}^{\prime}(x) & =0, \\
i Q_{1}^{\prime \prime}(x)-2 Q_{0}^{\prime}(x) Q_{2}^{\prime}(x)-\left[Q_{1}^{\prime}(x)\right]^{2}-V(x) & =0, \\
i Q_{n-1}^{\prime \prime}(x)-\sum_{j=0}^{n} Q_{j}^{\prime}(x) Q_{n-j}^{\prime}(x) & =0 \quad(n \geq 3) .
\end{aligned}
$$

The solution to these equations is

$$
\begin{aligned}
& Q_{0}(x)= \pm x \\
& Q_{1}(x)=0 \\
& Q_{2}(x)=\mp \frac{1}{2} \int_{0}^{x} d t V(t), \\
& Q_{3}(x)=-\frac{i}{4}[V(x)-V(0)] \\
& Q_{4}(x)= \pm \frac{1}{8}\left(V^{\prime}(x)-V^{\prime}(0)-\int_{0}^{x} d t V^{2}(t)\right), \\
& Q_{5}(x)=\frac{i}{16}\left[V^{\prime \prime}(x)-V^{\prime \prime}(0)-2 V^{2}(x)+2 V^{2}(0)\right], \\
& Q_{6}(x)=\mp \frac{1}{32}\left(V^{\prime \prime \prime}(x)-V^{\prime \prime \prime}(0)-5 V(x) V^{\prime}(x)+5 V(0) V^{\prime}(0)+\int_{0}^{x} d t\left[2 V^{3}(t)-V(t) V^{\prime \prime}(t)\right]\right),
\end{aligned}
$$

and so on. Note that $Q_{n}(x)$ is normalized so that $Q_{n}(0)=0$. In general, the formula for $Q_{n}^{\prime}(x)$ is

$$
Q_{n}^{\prime}(x)= \pm \frac{1}{2}\left[i Q_{n-1}^{\prime \prime}(x)-\sum_{j=1}^{n-1} Q_{j}^{\prime}(x) Q_{n-j}^{\prime}(x)\right] \quad(n \geq 3) .
$$

In order to obtain a WKB formula for the discriminant $\Delta(E)$ in Eq. (6) we need to evaluate $Q_{n}(x)$ at $x=P$. The periodicity of the potential $V(x)$ simplifies the results considerably; when $n$ is odd, $Q_{n}(P)=0$ and when $n$ is even, only the integrals in Eq. (12) remain:

$$
\begin{aligned}
& Q_{0}(P)= \pm P \\
& Q_{2}(P)=\mp \frac{1}{2} \int_{0}^{P} d t V(t), \\
& Q_{4}(P)=\mp \frac{1}{8} \int_{0}^{P} d t V^{2}(t), \\
& Q_{6}(P)=\mp \frac{1}{32} \int_{0}^{P} d t\left[2 V^{3}(t)-V(t) V^{\prime \prime}(t)\right],
\end{aligned}
$$

and so on.

The WKB formula for the discriminant is particularly simple when the potential $V(x)$ is $\mathcal{P} \mathcal{T}$ symmetric:

$$
\Delta(E)=2 \cos \left[\frac{1}{\epsilon} \sum_{n=0}^{\infty} \epsilon^{2 n} Q_{2 n}(P)\right] .
$$

One obtains the same formula for potentials that are real and symmetric under parity $\mathcal{P}$.

For the complex $\mathcal{P} \mathcal{T}$-symmetric potentials $V(x)$ in (8) the WKB formula for the discriminant in Eq. (15) is

$$
\Delta(E)=2 \cos \left[\frac{2 \pi}{\epsilon}+\frac{\Gamma(2 N+3 / 2) \sqrt{\pi}}{4(2 N+1) !} \epsilon^{3}+\frac{(2 N+1) \Gamma(2 N+1 / 2) \sqrt{\pi}}{32(2 N) !} \epsilon^{5}+\cdots\right] .
$$


A similar WKB formula exists for the real odd-parity potentials $V(x)=\sin ^{2 N+1}(x)$ :

$$
\Delta(E)=2 \cos \left[\frac{2 \pi}{\epsilon}-\frac{\Gamma(2 N+3 / 2) \sqrt{\pi}}{4(2 N+1) !} \epsilon^{3}-\frac{(2 N+1) \Gamma(2 N+1 / 2) \sqrt{\pi}}{32(2 N) !} \epsilon^{5}+\cdots\right] .
$$

We can illustrate the extreme accuracy of these WKB approximations by comparing them with numerical computations of the discriminant. For example, for $V(x)=i \sin ^{3}(x)$ at $\epsilon=0.2$ (which corresponds to $E=25$ ) we find numerically that $\Delta(25)=1.9999960002$. The first three orders of the WKB approximation taken from Eq. (16) give $\Delta(25)=2.0, \Delta(25)=1.9999961447$, and $\Delta(25)=1.9999960046$. Similarly, for $V(x)=\sin ^{3}(x)$ at $\epsilon=0.2$ we find numerically that $\Delta(25)=1.9999959937$. The first three orders of the WKB approximation taken from Eq. (17) give the same values: $\Delta(25)=2.0, \Delta(25)=1.9999961447, \Delta(25)=1.9999960046$.

Despite this impressive precision, the WKB formulas (16) and (17) cannot be used directly to answer the crucial question of whether there are band gaps because these approximations to the discriminant $\Delta(E)$ never cross the values \pm 2 . The reason for this inadequacy of the WKB approximation is that the differences $\left|\Delta_{\max }-2\right|$ and $\left|\Delta_{\min }+2\right|$ are exponentially small when $E>>1$. Therefore, these differences are subdominant with respect to the WKB asymptotic series and are not accessible to any order in powers of $\epsilon$. Indeed, the WKB series can only provide information about quantities with an algebraically small error, and not an exponentially small error. We emphasize that the WKB approximation has this shortcoming only at the maxima and minima of the approximation. At other points any exponential discrepancy is completely negligible compared with algebraic errors.

The WKB series is still an extremely useful ingredient in the numerical search for zeros of $\Delta(E) \pm 2$. (These zeros are the dividing points between bands and gaps.) Our procedure is first to find the energies at which there are maxima and minima of the WKB approximation to the discriminant and then to evaluate, with high numerical precision, the actual value of the discriminant in a tiny neighborhood of each of these points. By doing this we can determine whether or not the discriminant $\Delta(E)$ crosses the lines \pm 2 .

For the real periodic potentials $V(x)=\sin ^{2 N+1}(x)$ our procedure confirms the rigorous theoretical result that every maximum of the discriminant lies above 2 and every minimum lies below -2 . Consider, for example, the potential $V(x)=\sin (x)$. From Fig. 1, it is clear that the first maximum lies above 2. The first minimum occurs at $E=2.3138$, where the discriminant has the value -2.0038787 . The second maximum occurs at $E=4.0336$, where the discriminant is 2.000007. Similar behavior is found for the other potentials in the class $V(x)=\sin ^{2 N+1}(x)$. In stark contrast, for the potentials $V(x)=i \sin ^{2 N+1}(x)$, while the maxima of the discriminant lie above +2 , the minima of the discriminant lie above -2 . Thus, for these potentials there are no antiperiodic wave functions. As an example, lengthy and delicate numerical analysis verifies that for the potential $V(x)=i \sin (x)$ the first three maxima of the discriminant $\Delta(E)$ are located at $E=3.9664284, E=8.9857320$, and $E=15.99206621346$. The value of the discriminant $\Delta(E)$ at these energies is $2.000007,2.00000000000069$, and 2.000000000000000000015 . The first two minima of the discriminant are located at $E=2.1916$ and $E=6.229223$ and at these energies $\Delta(E)$ has the values -1.9953386 and -1.99999999527 . Similar behavior is found for the other potentials in the class (8).

We conclude by pointing out that from the expressions for $Q_{n}(P)$ in Eq. (14) the WKB series truncates if the potential is a polynomial in $e^{i x}$. For example, for the complex $\mathcal{P} \mathcal{T}$-symmetric periodic potential $V(x)=e^{i x}$ the WKB series in Eq. (15) truncates after the first term because $Q_{n}(2 \pi)$ vanishes for $n \geq 1$. For this case the WKB approximation is exact and the discriminant is given by

$$
\Delta(E)=2 \cos (2 \pi \sqrt{E}) .
$$

One can verify this result directly by solving the Schrödinger equation (1) for this potential exactly; the solution is a Bessel function: $\psi(x)=J_{2 \sqrt{E}}\left(2 e^{i x / 2}\right)$.

\section{ACKNOWLEDGEMENT}

We are grateful to the U.S. Department of Energy for financial support.

[1] C. Kittel, Introduction to Solid State Physics, 5th ed. (Wiley, New York, 1976). 
[2] W. Magnus and S. Winkler, Hill's Equation (Wiley, New York, 1966).

[3] E. Belokolos, A. Bobenko, V. Enol'skii, A. Its and V. Matveev, Algebro-Geometric Approach to Nonlinear Integrable Equations (Springer-Verlag, New York, 1994) and references therein.

[4] C. M. Bender and S. Boettcher, Phys. Rev. Lett. 80, 5243 (1998).

[5] C. M. Bender and S. Boettcher, J. Phys. A: Math. Gen. 31, L273 (1998).

[6] C. M. Bender and K. A. Milton, Phys. Rev. D 55, R3255 (1997).

[7] C. M. Bender and K. A. Milton, Phys. Rev. D 57, 3595 (1998).

[8] T. J. Hollowood, Nucl. Phys. B 386, 166(1992).

[9] D. R. Nelson and N. M. Shnerb, Phys. Rev. E 58, 1383 (1998).

[10] N. Hatano and D. R. Nelson, Phys. Rev. Lett. 77, 570 (1996), and Phys. Rev. B 56, 8651 (1997).

[11] C. M. Bender and S. A. Orszag, Advanced Mathematical Methods for Scientists and Engineers (McGraw-Hill, New York, 1978), Chap. 10. 